\title{
Effect of Short Foot Exercise on Quiet Stance for the Patients with Stroke
}

\author{
Paul Lee PT, $\mathrm{MPT}^{1}$, Byong-Yong Hwang PT, PhD ${ }^{* 2}$ \\ ${ }^{1}$ Dept. of Physical Therapy, Bobath memorial hospital \\ ${ }^{*}$ Dept. of Physical Therapy, Yongin University
}

Purpose Short foot exercise is a well-known treatment for foot intrinsic muscles. In this study, we investigated whether the addition of short foot exercise could enhance the effects of conventional physical therapy on quiet standing following a stroke. Methods Conventional therapeutic exercise group $(\mathrm{N}=17)$ was received conventional therapeutic exercise with physical therapists to increase the strength of affected lower limb for 6-weeks, 90 minutes/day, 5-day/week. Short foot exercise with conventional therapeutic exercise group $(\mathrm{N}=17)$ received conventional therapeutic exercise and short foot exercise with same intensity. The surface elapsed area was assessed by BioRescue (RM Ingenierie, France). Results There were no significant differences between conventional therapeutic exercise group and short foot exercise with conventional therapeutic exercise group in the surface elapsed area $(p>0.05)$. Conclusion Short Foot Exercise combined with conventional therapeutic exercise program appeared to show no benefit on quiet stance.

Key words Short foot exercise, Foot intrinsic muscles, Stroke, Static balance, Quiet stance

Corresponding author Byong-Yong Hwang (bobathkorea@hanmail.net)

Received date 18 January 2018

Revised date 8 February 2018

Accepted date 21 February 2018

\section{Introduction}

Balance is compensated in people with hemiplegia and hemiparesis. ${ }^{1)}$ Postural displacement for patients with hemiplegia can be difficult that of their age-matched peers. ${ }^{2)}$ Ability to maintain symmetric weight bearing is also challenged following a stroke, with patients bearing as much as $61 \%$ to $80 \%$ of their body weight through the non-affected lower limb. ${ }^{3)}$ Therefore, equal body weight bearing is key to their rehabilitation goal. Also, hemiplegia can cause an increment in the patient's surface elapsed area, which is defined as the distance of CoP trajectory on quiet standing. Recently, the 'short foot exercise' has been introduced to isolate contraction of the plantar intrinsic muscles. ${ }^{4)}$ There is an increasing evidence which suggests that training the foot intrinsic muscles via short foot exercise can improve one's balance. For example, reduced foot arch collapse and improved balance scores after the 4 weeks

doi : http:dx.doi.org/10.17817/2018.01.26.111232 of short foot exercise training in healthy individuals."

According to previous studies, a short foot exercise was the ideal method to facilitate the foot intrinsic muscles. Changed foot function through intrinsic' strengthening would be one of the options for restoring static balance for healthy individuals." However, previous studies were only done by healthy subjects. Up to $50 \%$ of hemiplegic patients suffer from foot disorders which decreases functional independence by decreased balance ability. ${ }^{7)}$ It is necessary to study the role of foot intrinsic muscles on static balance for the patients with stroke.

\section{Materials and Methods \\ 1) Subjects}

Our thirty-four subjects were the patients with hemiplegia secondary to stroke who had been referred by a physician for inpatient physical therapy evaluation and intervention. The subjects were randomly allocated into two groups of equal size by computer software program that generates the random sequence. 
Characteristics between CEG and SFE with CEG have the same base line before the beginning of study (Table 1). The primary inclusion criteria were that subjects have been diagnosed with hemiplegia from neurology and rehabilitation medicine specialist, a patient who were not relapsed back within 3-12 months from the onset of stroke, a patient who was able to walk 50-meter or to sit alone, a patient who had MAS grade lesser than 3, a patient who have not had any other neurological disease (eg. Peripheral neuropathy, parkinson's disease, dementia, etc.), surgical disease, or visual impairment, a patient who was able to respond properly from sensory stimuli by the examiner which was ranged score 24 points to 30 points in Korean version of Montreal Cognitive Assessment, and a patient who understood the content of study and agree to participate.

\section{2) Study design}

Total 34 participants assigned to an exercise program. The 17 participants randomly allocated to a conventional therapeutic exercise. The other 17 participants allocated to conventional and short foot exercise. However, the intensity of both groups was equal to 90 minutes. The participant was assessed at the baseline of pre-intervention and at the end of post-intervention, participated in a 6-week intervention period.

3) Outcome Measures
BioRescue (RM Ingenierie, France) allows to perform static analysis (footprints) and dynamic analysis by reproducing movements of the everyday life (get up, squat, move forward, move backward, make a step). Subjects will be asked to stand on sensor-plate within 30 degrees apart their feet. Prior to eye open and close instructions for static standing for 60 seconds each, participants were asked to stare front wall. Total elapsed distance and surface are will be evaluated in accordance with trajectory of center of gravity. ${ }^{8)}$

\section{4) Interventions}

1) Conventional therapeutic exercise group (CEG)

The established conventional therapeutic exercise had been applied. Specific four conventional therapeutic exercises for strengthening lower limb were performed: (1) manual lateralization of the patella, ${ }^{9)}$ (2) the pelvic bridging, ${ }^{10)}$ (3) rhythmic stabilization, ${ }^{11)}$ (4) quadriceps femoris muscle setting. ${ }^{12)}$ Total 90 minutes of conventional therapeutic exercise applied to CEG group.

2) Short foot exercise with conventional therapeutic exercise group (SFE + CEG)

Specific instructions were given to the subjects during SFE. During inhalation, subjects were instructed to contact the bottom of affected foot muscles and raise the arch without curling toes. Then, subjects were

Table 1. General characteristics of subjects.

\begin{tabular}{cccc} 
& CEG $(\mathrm{N}=17)$ & SFE with CEG (N=17) & $F / \chi^{2}$ \\
Gender (\%) & Male 13(76.4) & Male 8(47) & .170 \\
Age & Female 4 (23.6) & Female 9(53) & .992 \\
Height & $43.35 \pm 7.8$ & $42.64 \pm 8.78$ & .999 \\
Weight & $168.52 \pm 6.49$ & $170.00 \pm 5.11$ & .993 \\
MMSE & $66.00 \pm 7.94$ & $67.58 \pm 9.77$ & .414 \\
Onset & $25.47 \pm 1.48$ & $25.47 \pm 1.28$ & .846 \\
Hemiplegic & $10.41 \pm 2.39$ & $10.64 \pm 2.47$ & .452 \\
side (\%) & Left 6 (35.3) & Left 4(23.5) \\
\hline
\end{tabular}

Note. Values are mean \pm standard deviation.

SFE : Short Foot Exercise

CEG : Conventional Therapeutic Exercise Group 
asked to hold the arch for six seconds. During exhalation, the heightened arch was released to the ground. ${ }^{13)}$ Two surface EMG were placed on affected medio/lateral gastrocnemius muscles to measure EMG activity of extrinsic muscles in order to rule out extrinsic muscles substitution during SFE. Total 75 minutes of conventional therapeutic exercise and $15 \mathrm{mi}-$ nutes of SFE were applied to this group.

\section{5) Statistical analysis}

Study data were analyzed using the SPSS statistical package. Tests applied to the data included the Shapiro-Wilk test to determine whether data is normal and this assumption is met in data for the statistical test. Independent-sample t-test and paired t-tests allowed for comparisons between groups and within groups, respectively ( $p=0.05)$.

\section{Results}

There are no statistically significant differences in baseline homogeneity values between conventional therapeutic exercise group and short foot exercise with conventional therapeutic exercise group.

Both conventional therapeutic exercise group and short foot exercise with conventional therapeutic exercise group showed no significant improvements in static balance ability on Sa during quiet stance (from $2.63 \pm 4.00 \mathrm{~mm}^{2}, \quad 5.72 \pm 12.75 \mathrm{~mm}^{2}$ at baseline to $2.71 \pm 3.93 \mathrm{~mm}^{2}, 3.10 \pm 6.22 \mathrm{~mm}^{2}$ at post-intervention; $\mathrm{pa}=$ $0.533, \mathrm{pa}=0.148$ respectively). (Table 2 ).

\section{Discussion}

Maintaining balance when performing functional tasks is a complicated process that is influenced by a range of sensorimotor factors including lower-extremity muscle strength, and peripheral sensation. Also, there is a rising body of evidence to indicate that foot and ankle characteristics may affect the quality of balance and functional task. ${ }^{14)}$ Our major findings were that SFE combined with conventional therapeutic exercise did not enhance the effects of conventional therapeutic exercise on static balance in patients with hemiplegia secondary to stroke. Our findings are in general agreement with other researchers who reported intrinsic foot muscles are active in dynamic stance. In other words, intrinsic foot muscles play an active role in dynamic arch support, meaning they function only in dynamic stance. ${ }^{4)}$ Thus, static balance strongly relies on other factors rather than intrinsic muscles. This previous study supports a current finding that elapsed surface area in static standing, had no significant differences within a group as well as between the groups. Although there are many possible reasons for the lack of an effect in our study, the smallness of our sample size may have contributed to a type II statistical error, and further research with larger sample sizes can exclude that possibility.

\section{Conclusion}

Our results indicate that there was no benefit of conventional therapeutic exercise compared with foot intrinsic training when administered in combination with

Table 2. Comparison of baseline and post-intervention elapsed surface area between conventional therapeutic exercise group and short foot exercise with conventional therapeutic exercise group.

\begin{tabular}{|c|c|c|c|c|c|c|c|c|c|c|}
\hline & \multicolumn{4}{|c|}{ CEG ( $N=17)$} & \multicolumn{6}{|c|}{ SFE with CEG (N=17) } \\
\hline & Baseline & $\begin{array}{c}\text { Post- } \\
\text { Intervention }\end{array}$ & ta & pa & Baseline & $\begin{array}{c}\text { Post- } \\
\text { Intervention }\end{array}$ & ta & pa & tb & $p b$ \\
\hline $\begin{array}{l}\text { Surface area, } \\
\qquad\left(\mathrm{mm}^{2}\right)\end{array}$ & $2.63 \pm 4.00$ & $2.71 \pm 3.93$ & -.637 & .533 & $5.72 \pm 12.75$ & $3.10 \pm 6.22$ & 1.519 & .148 & 6.198 & .138 \\
\hline
\end{tabular}

Note. Values are mean \pm standard deviation, a within-group comparison, b between-group comparison, " $P<0.05, " P<0.001$

SFE : Short Foot Exercise, CEG : Conventional Therapeutic Exercise Group 
conventional therapeutic exercise on static stance when provided 90 minutes over a 6-week period to inpatients with hemiplegia secondary to stroke. The results of this study may support that intrinsic muscles activation has no validation in ipsilateral side of the limb on quiet stance. This factor, along with evidence for the neuromuscular effect of SFE, suggest that foot function and foot intrinsic muscles may be interrelated on dynamic stance and that this relationship needs to be investigated further.

\section{References}

1. Bohannon RW. Gait performance of hemiparetic stroke patients: selected variab les. Arch Phys Med Rehabil. 1987;68:777-81

2. Nichols DS. Balance retraining after stroke using force platform biofeedback. Phys Ther. 1997;77:553-558

3. Sackley CM, Baguly BI. Visual feedback after stroke with balance performance monitor: two single case studies. Clin Rehabil. 1993;7:189-95

4. Janda V, Vavrova M, Hervenova A, et al. Sensory motor stimulation. in: C. Liebenson (Ed.) Rehabilitation of the spine: a practitioner's manual. 2nd edition. Lippincott Williams \& Wilkins, Baltimore, MD; 2006:517-9.

5. Mulligan EP, Cook PG. Effect of plantar intrinsic muscle training on medial longitudinal arch morphology and dynamic function. Man Ther. 2013;18(5):425-30.

6. Mann R, Inman VT. Phasic activity of intrinsic muscles of the foot. The Journal of Bone and Joint Sturger. 1964;46(3):469-81

7. Patrick JH, Keenan MAE. Gait analysis to assist walking after stroke. The Lancet. 2007;369(9558):256-7

8. LEE J, Seo K. The effects of stair walking training on the balance ability of chronic stroke patients. Phys Ther. 2014;26(4):517

9. Douciette SA, Goble EM. The effect of exercise on patellar tracking in lateral patellar compression syndrome. Am J Sports Med. 1992;20(4):434-40.

10. Bahr R, Mæhlum S. Clinical guide to sports injuries. Human Kinetics. 2004.

11. Adler SS, Beckers D, Buck M. PNF in Practice. 1993

12. Soderberg GL, Minor SD, Arnold $K$, et al. Electromyographic analysis of knee exercises in healthy subjects and in patients with knee pathologies. Phys Ther. 1987;67(11):1691-6.

13. Newsham KR. Functional Rehabilitation: Strengthening the Intrinsic Foot Muscles. IJATT. 2010;15(1).

14. Menz HB, Morris ME, Lord SR. Foot and ankle characteristics associated with impaired balance and functional ability in older people. J Gerontol Seires A. 2005;60 (12),1546-52. 